\title{
LA DANZA EN PERSONAS CON DISCAPACIDAD INTELECTUAL: REVISIÓN SISTEMÁTICA E IDENTIFICACIÓN DE LOS ELEMENTOS NECESARIOS PARA EVALUAR CAMBIOS DE MOVIMIENTO*
}

Silvia Barnet López

Universitat Ramon Llull, FPCEE Blanquerna

\section{Susana Pérez Testor}

Universitat Ramon Llull, FPCEE Blanquerna

\section{Mar Arbonés Garcia}

Universitat Autònoma de Barcelona

\author{
Myriam Guerra Balic \\ Universitat Ramon Llull, FPCEE Blanquerna
}

\section{Resumen}

La danza se considera una herramienta que contribuye al bienestar de la persona desde una perspectiva integradora. Estudios anteriores afirman, a través del Dibujo de la Figura Humana (DFH), que mejora el bienestar emocional y físico en población con Discapacidad Intelectual (DI). El DFH evalúa el antes y el después de la sesión de danza, pero no durante. Así pues, los objetivos de este estudio son: Actualizar estudios anteriores sobre el test del DFH en población con DI que participan en actividades de danza. Revisar el tipo de instrumento más adecuado para población con DI que complemente la información que nos ofrece el test DFH, en relación a los cambios de movimiento que suceden durante la sesión de danza. Determinar las características que debería tener el protocolo para la creación del instrumento de evaluación de los cambios de movimiento.

\section{Palabras-clave: DANZA; DISCAPACIDAD INTELECTUAL; EVALUACIÓN}

\section{Abstract}

From a holistic approach, the dance is considered a tool which contributes to the wellbeing of a person. Previous studies claimed to improve the emotional and physical well-being through the Human Figure Drawing (HFD) on a population with intellectual disability (ID). The HFD was evaluated before and after a dance session, but not throughout it. Therefore, the objectives of this study are: update previous studies of the HFD test on a ID population participating in dance activities. To review the most appropriate type of instrument for people with ID to supplement the information obtained from the HFD test related to the changes in motion that occur during the dance session. Determinate which characteristics the protocol should have in order to generate an evaluation tool to assess movement changes.

Keywords: DANCE; INTELLECTUAL DISABILITY; EVALUATION

\section{Barnet López, Silvia, Susana Pérez Testor, Mar Arbonés García \& Myriam Guerra Balic. 2015. "Instrumentos de evaluación en la danza con personas con discapacidad intelectual”. AusArt 3 (1): 213-227. D0I: 10.1387 /ausart. 14398}

\footnotetext{
* Proyecto parcialmente financiado por el Ministerio de Economía y Competitividad (I+D DEP 2012-38984) y por la Beca de Recerca Blanquerna de la FPCEE-Blanquerna, Universitat Ramón Lull.
}

\section{AUSART}




\section{INTRODUCCIÓN}

\section{1. Danza y movimiento en población con discapacidad intelec- TUAL}

La danza es reconocida como tratamiento efectivo por la American Dance Therapy Association en personas con discapacidades médicas, sociales, físicas o psicológicas (ADTA, 2011) desde la perspectiva de la Danza Movimiento Terapia (DMT). El movimiento es inherente a la persona, éste adquiere protagonismo a raíz de las aportaciones del análisis del movimiento de Rudolf Laban (1987). Cuando se habla de movimiento se incluye; la forma de caminar, de gesticular, de relacionarnos con los demás, de establecer contacto físico o de evitarlo, entre otros elementos. Con el conjunto de estos elementos es posible determinar un perfil de movimiento (Fischman 2008).

La DMT, junto con otras terapias creativas como la arte terapia o músico terapia, son consideradas efectivas en la intervención en la Discapacidad Intelectual (DI). Dan espacio a la expresión más libre del sujeto, sin forzar un razonamiento previo, permitiendo establecer una conexión terapeuta-paciente más próxima, así como la del paciente consigo mismo (Hurley, Tomasulo y Pfadt 1998).

En la actualidad la asociación para personas con síndrome de Down y otras discapacidades intelectuales (ASSIDO), emplea la danza y la música, entre otras vertientes del arte, para el bienestar y la mejora de la calidad de vida en personas con DI (Ballesta, Onil y Mesas 2011).

En DI estamos trabajando con grupos heterogéneos con un amplio abanico de niveles de funcionamiento, de limitaciones y de cualidades. Por lo tanto, consideramos que el diagnóstico proporciona una parte de la información, pero para la intervención y la evaluación deberemos tener presente las características individuales (Jané, 2004).

\subsection{Evaluación en POBlación CON Discapacidad intelectual}

Se han realizado estudios para evaluar la eficacia de la DMT en población con $\mathrm{DI}$, los cuales muestran cambios positivos a nivel psicológico y físico, al inicio y al final de una sesión o de un proceso terapéutico. Las variables que se tuvieron en cuenta fueron las habilidades físicas de movimiento, como 
la coordinación; la autonomía personal; la satisfacción y goce personal; y la imagen corporal (Hecox, Levine y Scott 1975; Rogers 1977; Tipple 1975).

Meredith Ritter y Kathryn Low (1996) incluyen en su meta-análisis una valoración de estudios cuantitativos en DMT en diferentes poblaciones. Se observan cómo limitaciones, estudios con una muestra pequeña y pocos estudios en población adulta con DI.

Otro meta-análisis es el realizado por Sabine Koch, Teresa Kunz, Sissy Lykou y Robin Cruz (2014) con el objetivo de determinar los efectos de la DMT y el uso terapéutico de la danza desde 1996. Se observa que la mayoría de estudios están enfocados al espectro del autismo en población infantil. Las conclusiones constatan que tras la aplicación del programa, los pacientes mejoran en humor, afecto, calidad de vida, imagen corporal y competencia interpersonal.

Estudios anteriores (Pérez-Testor et al. 2009; Martín, Pérez-Testor y Guerra 2009; Pérez-Testor et al. 2012; Barnet y Guerra 2012; Barnet, Pérez Testor y Guerra 2013; Pérez-Testor y Griñó 2014) muestran los beneficios que aporta la danza a través de la evaluación del Dibujo de la Figura Humana (DFH). El DFH es un test utilizado en distintas poblaciones, principalmente en niños y personas con dificultades de expresión oral (Koppitz 2000). El movimiento, al igual que el dibujo, son vehículos de expresión de emociones y sentimientos del pensamiento consciente e inconsciente de la persona (Fischman 2008). Rudolf Laban (1987) propone identificar patrones comunes en poblaciones con características similares y crea la Teoría del Análisis del Movimiento (AML), para describir y analizar el movimiento humano. La utilización del DFH nos permite evaluar el cambio de la persona, antes y después de la sesión de danza, tanto a nivel evolutivo como a nivel emocional, pero no durante la sesión.

Según Teresa Anguera (1989) si el problema se refiere a una conducta compleja que se desarrolla en el entorno habitual de la persona, el método observacional será una herramienta útil para poder desarrollar un estudio sistematizado y cuantitativo. El sistema de categorías como instrumento de medida observacional nos permitirá precisión y objetividad.

Las investigaciones fundamentadas en la observación son útiles sobretodo para aquellas poblaciones que presentan dificultades para proporcionar informes verbales por algún tipo de limitación, como por ejemplo en personas con DI (Anguera 1989). 


\section{OBJETIVOS DEL ESTUDIO}

Los objetivos del estudio son: (1) Actualizar estudios anteriores sobre el test del DFH en población con DI que participan en actividades de danza. (2) Revisar el tipo de instrumento más adecuado para población con DI que complemente la información que nos ofrece el test DFH, en relación a los cambios de movimiento que suceden durante la sesión de danza. (3) Determinar las características que debería tener el protocolo para la creación del instrumento de evaluación de los cambios de movimiento.

\section{METODOLOGÍA}

Se realizaron dos revisiones sistemáticas de la literatura científica publicada en los últimos 10 años según la propuesta de Verdonschot, de Witte, Reichrath, Buntinx y Curfs (2009), en el que se puntúan todos los artículos en un procedimiento multietápico.

Primera revisión: Estudios que fueron recuperados en las bases de datos: ERIC, Psycinfo y Pubmed, con las siguientes palabras clave: Intellecutal Disabilities, Intellectual Disability, Developmental Disabilities, Mental Retardation y/o Dance or Dance Therapy y/o Human Figure Drawing (ver Tabla 1).

Segunda revisión: Estudios recuperados en las bases de datos: ERIC, Psycinfo y Pubmed, con las siguientes palabras clave: Intellecutal Disabilities, Intellectual Disability, Developmental Disabilities, Mental Retardation y/o movement analysis y/o evaluation (ver Tabla 2).

Para ambas revisiones sistemáticas se determinaron los siguientes criterios de inclusión y de exclusión:

Criterios de Inclusión- Para la primera revisión sistemática se incluyeron aquellos artículos sobre población con DI; que relacionen la DI con danza o elementos de la danza terapia o de terapias creativas, o estudios con población con DI donde se utilice el instrumento del DFH. También se incluirán aquellos artículos que relacionen los tres elementos la DI, la Danza/Danza Terapia y el DFH. En el caso de la segunda revisión sistemática se incluyen artículos sobre 
población con $\mathrm{DI}$; que relacionen la DI con tipos o instrumentos de evaluación o análisis del movimiento. También se incluyeron aquellos artículos que relacionen los tres elementos la DI y la evaluación y el análisis del movimiento.

Criterios de Exclusión- Este criterio es común para ambas revisiones, no se aceptaran aquellos artículos publicados hace más de 10 años.

\section{Tabla 1.}

Artículos extraídos de la revisión sistemática de estudios con población con DI sobre el test del DFH en sesiones de danza.

Artículos extraídos de las bases de datos que reúnen los criterios de inclusión.

$$
\mathrm{N}=16 \text { artículos }
$$

Total de artículos que reúnen los criterios de inclusión.

$$
\mathrm{N}=17 \text { artículos }
$$

Tabla 2.

Artículos extraídos de la revisión sistemática de estudios con población con DI instrumentos de evaluación en sesiones de danza.

Artículos extraídos de las bases de datos que reúnen los criterios de inclusión.

$$
N=14 \text { artículos }
$$

Artículos adicionales extraídos de fuentes originales que reúnen los criterios de inclusión.

$$
\mathrm{N}=1 \text { artículo }
$$

$\mathrm{N}=14$ artículos

Artículos adicionales extraídos de fuentes originales que reúnen los criterios de inclusión.

$$
\mathrm{N}=2 \text { artículos }
$$

Total de artículos que reúnen los criterios de inclusión.

$$
\mathrm{N}=16 \text { artículos }
$$

Posteriormente a las dos revisiones sistemáticas, se extrajeron aquellos elementos importantes para nuestro estudio y vinculados con el marco teórico; 
para determinar las características de una propuesta de protocolo para la realización de un instrumento de evaluación para sesiones de danza en DI.

\section{RESULTADOS}

En la primera revisión se seleccionaron un total de 17 artículos vinculados a estudios con población con DI sobre el test del DFH en sesiones de danza (ver Tabla 3).

\section{Tabla 3.}

Resultados obtenidos de 19 estudios que cumplen los criterios de inclusión y exclusión para la primera revisión sistemática.

\begin{tabular}{|c|c|c|c|c|}
\hline $\begin{array}{l}\text { Autor y año } \\
\text { publicación }\end{array}$ & Título & Diagnóstico & Instrumentos & $\begin{array}{c}\text { Temática o } \\
\text { Intervención }\end{array}$ \\
\hline $\begin{array}{l}\quad 2006 \\
\text { Jobling A., } \\
\text { Virji-Babul, } \\
\text { N., y Nichols } \\
\text { D. }\end{array}$ & $\begin{array}{l}\text { Children with } \\
\text { Down Syndrome: } \\
\text { Discovering the } \\
\text { Joy of Movement }\end{array}$ & $\begin{array}{l}\text { Niños con } \\
\text { síndrome de } \\
\text { Down }\end{array}$ & $\begin{array}{l}\text { Observación } \\
\text { sesiones: } \\
\text { Movimientos }\end{array}$ & $\begin{array}{l}\text { Danza y } \\
\text { movimiento }\end{array}$ \\
\hline $\begin{array}{l}2006 \\
\text { Lee, A., y } \\
\text { Hobson, P. }\end{array}$ & $\begin{array}{l}\text { Drawing Self and } \\
\text { Others: How Do } \\
\text { Children with } \\
\text { Autism Differ } \\
\text { from Those } \\
\text { with Learning } \\
\text { Difficulties? }\end{array}$ & $\begin{array}{l}\text { Niños y } \\
\text { adolescentes } \\
\text { con Autismo }\end{array}$ & $\begin{array}{l}\text { Dibujo de } \\
\text { la figura } \\
\text { humana }\end{array}$ & - \\
\hline $\begin{array}{l}\quad 2006 \\
\text { Stepshenson, } \\
\text { J. }\end{array}$ & $\begin{array}{l}\text { Music Therapy } \\
\text { and the Education } \\
\text { of Students } \\
\text { with Severe } \\
\text { Disabilities }\end{array}$ & $\begin{array}{l}\text { Estudiantes } \\
\text { con DI severa } \\
\text { y moderada }\end{array}$ & - & $\begin{array}{l}\text { Músico } \\
\text { Terapia }\end{array}$ \\
\hline
\end{tabular}




\begin{tabular}{|c|c|c|c|c|}
\hline $\begin{array}{l}\quad 2007 \\
\text { Wheeles, } \\
\text { B.L., y Stultz, } \\
\text { S. }\end{array}$ & $\begin{array}{l}\text { Using } \\
\text { Typical Infant } \\
\text { Development } \\
\text { to Inform Music } \\
\text { Therapy with } \\
\text { Children with } \\
\text { Disabilities }\end{array}$ & $\begin{array}{l}\text { Niños con } \\
\text { problemas de } \\
\text { desarrollo y } \\
\text { atención }\end{array}$ & $\begin{array}{l}\text { Análisis de la } \\
\text { Observación } \\
\text { a través de } \\
\text { las sesiones } \\
\text { registradas } \\
\text { basado en } \\
\text { el modelo de } \\
\text { desarrollo } \\
\text { psicosocial } \\
\text { de } \\
\text { Greensapn }\end{array}$ & $\begin{array}{l}\text { Músico } \\
\text { Terapia }\end{array}$ \\
\hline $\begin{array}{l}\quad 2008 \\
\text { Hui Keow, L., } \\
\text { y Slaughter } \\
\text { V. }\end{array}$ & $\begin{array}{l}\text { Brief Report: } \\
\text { Human Figure } \\
\text { Drawings by } \\
\text { Children with } \\
\text { Asperger's } \\
\text { Syndrome }\end{array}$ & $\begin{array}{l}\text { Niños con } \\
\text { síndrome de } \\
\text { Asperger }\end{array}$ & $\begin{array}{l}\text { Dibujo Libre, } \\
\text { Dibujo de } \\
\text { la Figura } \\
\text { Humana } \\
\text { y Test: } \\
\text { persona, } \\
\text { casa y árbol }\end{array}$ & - \\
\hline $\begin{array}{l}\qquad 2008 \\
\text { Nicole Martin, } \\
\text { Lawrence, } \\
\text { K.S. }\end{array}$ & $\begin{array}{l}\text { Assessing Portrait } \\
\text { Drawings Created } \\
\text { by Children and } \\
\text { Adolescents With } \\
\text { Autism Spectrum } \\
\text { Disorder }\end{array}$ & $\begin{array}{l}\text { Adolescentes } \\
\text { con espectro } \\
\text { autista }\end{array}$ & $\begin{array}{l}\text { Dibujos de } \\
\text { rostros }\end{array}$ & - \\
\hline $\begin{array}{c}2008 \\
\text { Skoning, S.N. }\end{array}$ & $\begin{array}{l}\text { Movement and } \\
\text { Dance Inclusive } \\
\text { Classroom }\end{array}$ & $\begin{array}{l}\text { Estudiantes } \\
\text { con DI o } \\
\text { trastornos } \\
\text { emocionales } \\
\text { o trastorno } \\
\text { por déficit } \\
\text { de atención, } \\
\text { otros }\end{array}$ & $\begin{array}{l}\text { Propuesta } \\
\text { de Teoría: } \\
\text { Análisis de } \\
\text { Observación } \\
\text { del } \\
\text { Movimiento } \\
\text { de Rudolf } \\
\text { Laban }\end{array}$ & $\begin{array}{l}\text { Movimiento } \\
\text { Creativo y } \\
\text { Danza }\end{array}$ \\
\hline $\begin{array}{l}\quad 2009 \\
\text { Lister, S., } \\
\text { Denise, T., } \\
\text { Snow, S., et } \\
\text { al. }\end{array}$ & $\begin{array}{l}\text { Development of } \\
\text { a creative arts } \\
\text { therapies center } \\
\text { for people with } \\
\text { developmental } \\
\text { disabilities. }\end{array}$ & DI & - & $\begin{array}{l}\text { Terapias } \\
\text { Creativas }\end{array}$ \\
\hline $\begin{array}{l}\quad 2010 \\
\text { Krikeli, V., } \\
\text { Michailidis, A., } \\
\text { y Klavdianou, } \\
\text { N.D. }\end{array}$ & $\begin{array}{l}\text { Communication } \\
\text { improvement } \\
\text { through music: } \\
\text { The case of } \\
\text { children with } \\
\text { developmental } \\
\text { disabilities }\end{array}$ & $\begin{array}{l}\text { Niños con } \\
\text { discapaci- } \\
\text { dades en el } \\
\text { desarrollo }\end{array}$ & $\begin{array}{l}\text { State-Trait } \\
\text { Anxiety } \\
\text { Inventory } \\
\text { Scale }\end{array}$ & $\begin{array}{l}\text { Músico } \\
\text { Terapia }\end{array}$ \\
\hline
\end{tabular}




\begin{tabular}{|c|c|c|c|c|}
\hline $\begin{array}{l}\quad 2011 \\
\text { Betty, B., y } \\
\text { Peggy, V. }\end{array}$ & $\begin{array}{l}\text { The Adapted } \\
\text { Dance Process: } \\
\text { Planning, } \\
\text { Partnering, and } \\
\text { Performing }\end{array}$ & Discapacidad & - & $\begin{array}{l}\text { Programa } \\
\text { de } \\
\text { pedagogía } \\
\text { de la danza } \\
\text { inclusivo }\end{array}$ \\
\hline $\begin{array}{c}2011 \\
\text { Johnnye, K. }\end{array}$ & $\begin{array}{l}\text { Camp } \\
\text { Thunderbird: } \\
\text { Taking Flight } \\
\text { with Dance } \\
\text { and Physical } \\
\text { Education } \\
\text { for Special } \\
\text { Populations }\end{array}$ & $\begin{array}{l}\text { Espectro } \\
\text { Autista, } \\
\text { Retraso } \\
\text { Mental, } \\
\text { Trastornos del } \\
\text { comporta- } \\
\text { miento o } \\
\text { emocionales, } \\
\text { otros }\end{array}$ & - & $\begin{array}{l}\text { Programa } \\
\text { de } \\
\text { promoción } \\
\text { de la } \\
\text { actividad } \\
\text { física y las } \\
\text { artes; danza } \\
\text { y música }\end{array}$ \\
\hline $\begin{array}{l}\qquad 2011 \\
\text { Kilbane, A., y } \\
\text { Johaba, A. }\end{array}$ & $\begin{array}{l}\text { Therapy } \\
\text { Expectations: } \\
\text { Preliminary } \\
\text { Exploration and } \\
\text { Measurement } \\
\text { in Adults with } \\
\text { Intellectual } \\
\text { Disabilities }\end{array}$ & $\begin{array}{l}\text { Adultos con } \\
\text { DI }\end{array}$ & $\begin{array}{l}\text { Psychometric } \\
\text { evaluation of } \\
\text { the therapy } \\
\text { expectation } \\
\text { measure } \\
\text { (TEAM) (test } \\
\text { - retest) and } \\
\text { semi- } \\
\text { structured } \\
\text { interviews }\end{array}$ & - \\
\hline $\begin{array}{l}\qquad 2012 \\
\text { Emck, C., } \\
\text { Maaike, P., y } \\
\text { Manon, V. }\end{array}$ & $\begin{array}{l}\text { Body experience } \\
\text { in children with } \\
\text { intellectual } \\
\text { disabilities with } \\
\text { and without } \\
\text { externalising } \\
\text { disorders. }\end{array}$ & Niños con DI & $\begin{array}{l}\text { Un } \\
\text { cuestionario } \\
\text { para niños } \\
\text { sobre la } \\
\text { experiencia } \\
\text { corporal }\end{array}$ & $\begin{array}{l}\text { Terapia } \\
\text { psicomotriz }\end{array}$ \\
\hline $\begin{array}{l}\quad 2012 \\
\text { Tsimaras, } \\
\text { V.K., } \\
\text { Giamouridou } \\
\text { G.A., } \\
\text { Kokaridas } \\
\text { D.G. et. al. }\end{array}$ & $\begin{array}{l}\text { The effect of a tra- } \\
\text { ditional dance tra- } \\
\text { ining program on } \\
\text { dynamic balance } \\
\text { of individuals with } \\
\text { mental retarda- } \\
\text { tion. }\end{array}$ & $\begin{array}{l}\text { Retraso } \\
\text { Mental: Leve } \\
\text { o moderado }\end{array}$ & $\begin{array}{l}\text { Pruebas de } \\
\text { esfuerzo: } \\
\text { Equilibrio } \\
\text { dinámico }\end{array}$ & $\begin{array}{l}\text { Programa } \\
\text { de danza } \\
\text { tradicional } \\
\text { Griega }\end{array}$ \\
\hline
\end{tabular}




\begin{tabular}{|c|c|c|c|c|}
\hline $\begin{array}{r}2013 \\
\text { Amos, P. }\end{array}$ & $\begin{array}{l}\text { Rhythm and } \\
\text { timing in autism: } \\
\text { Learning to } \\
\text { dance. }\end{array}$ & Autismo & - & Danza \\
\hline $\begin{array}{l}\quad 2014 \\
\text { Koch, S., } \\
\text { Mehl, L., } \\
\text { Sobanski, E., } \\
\text { et al. }\end{array}$ & $\begin{array}{l}\text { Fixing the mirrors: } \\
\text { A feasibility study } \\
\text { of the effects of } \\
\text { dance movement } \\
\text { therapy on young } \\
\text { adults with autism } \\
\text { spectrum disorder }\end{array}$ & $\begin{array}{l}\text { Adultos } \\
\text { jóvenes con } \\
\text { Autismo }\end{array}$ & - & $\begin{array}{l}\text { Danza } \\
\text { Movimiento } \\
\text { Terapia }\end{array}$ \\
\hline $\begin{array}{l}\quad 2015 \\
\text { Teo, J., y Ong } \\
\text { Puay, H. }\end{array}$ & $\begin{array}{l}\text { Dance and } \\
\text { movement as } \\
\text { therapy for } \\
\text { children with } \\
\text { autism spectrum } \\
\text { disorders } \\
\text { (ASD): A case } \\
\text { for Kuching, } \\
\text { Sarawak. }\end{array}$ & Autismo & - & $\begin{array}{l}\text { Danza } \\
\text { Movimiento } \\
\text { Terapia }\end{array}$ \\
\hline
\end{tabular}

En relación a la segunda revisión sistemática, se seleccionaron un total de 16 artículos referentes a estudios vinculados a instrumentos de evaluación para sesiones de danza en población con DI (ver Tabla 4).

Tabla 4.

Resultados obtenidos de 16 estudios que cumplen los criterios de inclusión y exclusión para la segunda revisión sistemática.

\begin{tabular}{|c|c|c|c|}
\hline $\begin{array}{l}\text { Autor y año } \\
\text { publicación }\end{array}$ & Título & Diagnóstico & Instrumentos \\
\hline $\begin{array}{l}\quad 2007 \\
\text { Martin, L., } \\
\text { Hirdes, J., } \\
\text { Fries, E. y } \\
\text { Smith, T. }\end{array}$ & $\begin{array}{l}\text { Development and } \\
\text { Psychometric Properties } \\
\text { of an Assessment for } \\
\text { Persons with Intellectual } \\
\text { Disability-The InterRAI ID }\end{array}$ & DI & $\begin{array}{l}\text { InterRAI-Intellectual } \\
\text { Disability } \\
\text { Dementia } \\
\text { Questionnaire } \\
\text { Reiss Screen }\end{array}$ \\
\hline $\begin{array}{l}\quad 2008 \\
\text { Starr,J.y } \\
\text { Marsden, L. }\end{array}$ & $\begin{array}{l}\text { Characterisation of user- } \\
\text { defined health status } \\
\text { in older adults with } \\
\text { intellectual disabilities }\end{array}$ & $\begin{array}{l}\text { Adultos } \\
\text { mayores con } \\
\text { DI }\end{array}$ & $\begin{array}{l}\text { User-led health } \\
\text { assessment }\end{array}$ \\
\hline
\end{tabular}




\begin{tabular}{|c|c|c|c|}
\hline $\begin{array}{l}\quad 2009 \\
\text { Bossaert, G., } \\
\text { Kuppens, S., } \\
\text { Buntinx, W., } \\
\text { et al. }\end{array}$ & $\begin{array}{l}\text { Usefulness of the } \\
\text { Supports Intensity Scale } \\
\text { (SIS) for Persons with } \\
\text { Other than Intellectual } \\
\text { Disabilities }\end{array}$ & DI & $\begin{array}{l}\text { Supports Intensity } \\
\text { Scale (SIS) }\end{array}$ \\
\hline $\begin{array}{l}\qquad 2009 \\
\text { Matson, J., } \\
\text { Dempsey, T. } \\
\text { y LoVullo, S. }\end{array}$ & $\begin{array}{l}\text { Characteristics of social } \\
\text { skills for adults with } \\
\text { intellectual disability, } \\
\text { autism and PDD-NOS }\end{array}$ & $\begin{array}{l}\text { Adultos con DI, } \\
\text { autismo y otros }\end{array}$ & $\begin{array}{l}\text { Matson Evaluation } \\
\text { of Social Skills for } \\
\text { undividuals with } \\
\text { severe retardation } \\
\text { (MESSIER) }\end{array}$ \\
\hline $\begin{array}{l}\quad 2009 \\
\text { Singh, A., } \\
\text { Matson, J.; } \\
\text { Mouttapa, } \\
\text { M., Pella, R., } \\
\text { et al. }\end{array}$ & $\begin{array}{l}\text { A Critical Item Analysis of } \\
\text { the QABF: Development } \\
\text { of a Short Form } \\
\text { Assessment Instrument }\end{array}$ & DI & $\begin{array}{l}\text { Questions About } \\
\text { Behavioral Function } \\
\text { (QABF) }\end{array}$ \\
\hline $\begin{array}{c}2009 \\
\text { Woodard, C. }\end{array}$ & $\begin{array}{l}\text { Psychometric Properties } \\
\text { of the ASPeCT-DD: } \\
\text { Measuring Positive } \\
\text { Traits in Persons } \\
\text { with Developmental } \\
\text { Disabilities }\end{array}$ & DI & $\begin{array}{l}\text { Assessment } \\
\text { Scale for Positive } \\
\text { Character Traits- } \\
\text { Developmental } \\
\text { Disabilities } \\
\text { (ASPeCT-DD) }\end{array}$ \\
\hline $\begin{array}{l}\quad 2010 \\
\text { Willner, P., } \\
\text { Bailey, R., } \\
\text { Parry, R. y } \\
\text { Dymond, S. }\end{array}$ & $\begin{array}{l}\text { Evaluation of executive } \\
\text { functioning in people with } \\
\text { intellectual disabilities }\end{array}$ & DI & $\begin{array}{l}\text { Behavioural } \\
\text { Assessment of } \\
\text { the Dysexecutive } \\
\text { Syndrome } \\
\text { (BADS-C) y } \\
\text { Cambridge } \\
\text { Executive } \\
\text { Functioning } \\
\text { Assessment (CEFA) }\end{array}$ \\
\hline $\begin{array}{l}\quad 2011 \\
\text { Kilbane, A. y } \\
\text { Jahoda, A. }\end{array}$ & $\begin{array}{l}\text { Therapy Expectations: } \\
\text { Preliminary Exploration } \\
\text { and Measurement in } \\
\text { Adults with Intellectual } \\
\text { Disabilities }\end{array}$ & Adultos con DI & $\begin{array}{l}\text { Therapy expectation } \\
\text { measure (TEAM) } \\
\text { Semi-structured } \\
\text { interviews }\end{array}$ \\
\hline
\end{tabular}




\begin{tabular}{|c|c|c|c|}
\hline $\begin{array}{l}\quad 2012 \text { ' } \\
\text { McKenzie, K., } \\
\text { Paxton, D., } \\
\text { Murray, G., } \\
\text { et al. }\end{array}$ & $\begin{array}{l}\text { The Evaluation of } \\
\text { a Screening Tool } \\
\text { for Children with an } \\
\text { Intellectual Disability: The } \\
\text { Child and Adolescent } \\
\text { Intellectual Disability } \\
\text { Screening Questionnaire }\end{array}$ & $\begin{array}{l}\text { Niños y } \\
\text { adolescentes } \\
\text { con DI }\end{array}$ & $\begin{array}{l}\text { Child and } \\
\text { Adolescent } \\
\text { Intellectual } \\
\text { Disability Screening } \\
\text { Questionnaire" } \\
\text { (CAIDS-Q) }\end{array}$ \\
\hline $\begin{array}{l}\qquad 2012 \\
\text { Vakil, E. y } \\
\text { Lifshiltz- } \\
\text { Zehavi, H. }\end{array}$ & $\begin{array}{l}\text { Solving the Raven } \\
\text { Progressive Matrices by } \\
\text { Adults with Intellectual } \\
\text { Disability with/without } \\
\text { Down Syndrome: } \\
\text { Different Cognitive } \\
\text { Patterns as Indicated by } \\
\text { Eye-Movements }\end{array}$ & $\begin{array}{l}\text { Adultos con } \\
\text { síndrome de } \\
\text { Down o DI no } \\
\text { especificado y } \\
\text { niños con TD }\end{array}$ & Matrices de Raven \\
\hline $\begin{array}{l}\quad 2013 \\
\text { Chaplin, E., } \\
\text { Chester, R., } \\
\text { Tsakanikos, } \\
\text { E., et al. }\end{array}$ & $\begin{array}{l}\text { Reliability and Validity } \\
\text { of the SAINT: A Guided } \\
\text { Self-Help Tool for } \\
\text { People with Intellectual } \\
\text { Disabilities }\end{array}$ & Adultos con DI & $\begin{array}{l}\text { Self Assessment } \\
\text { and Intervention } \\
\text { (SAINT) } \\
\text { Glasgow } \\
\text { Depression Scale- } \\
\text { Learning Disabilities } \\
\\
\text { Glasgow Anxiety } \\
\text { Scale- Intellectual } \\
\text { Disabilities }\end{array}$ \\
\hline $\begin{array}{r}2013 \\
\text { Gies, M. }\end{array}$ & $\begin{array}{l}\text { The use video prompting } \\
\text { on the acquisition, } \\
\text { maintenance, and } \\
\text { generalization of a line } \\
\text { dance by adolescents } \\
\text { with autism spectrum } \\
\text { disorders }\end{array}$ & $\begin{array}{l}\text { Adolescentes } \\
\text { con autismo }\end{array}$ & Modelado en video \\
\hline $\begin{array}{l}\qquad 2013 \\
\text { McVilly, K., } \\
\text { Webber, L., } \\
\text { Sharp, G. y } \\
\text { et al. }\end{array}$ & $\begin{array}{l}\text { The Content Validity of } \\
\text { the Behaviour Support } \\
\text { Plan Quality Evaluation } \\
\text { Tool (BSP-QEII) and its } \\
\text { Potential Application in } \\
\text { Accommodation and } \\
\text { Day-Support Services for } \\
\text { Adults with Intellectual } \\
\text { Disability }\end{array}$ & Adultos con DI & $\begin{array}{l}\text { Behaviour support } \\
\text { plans (BSPs) y Plan } \\
\text { Quality Evaluation } \\
\text { tool (BSP-QEII) }\end{array}$ \\
\hline
\end{tabular}




\begin{tabular}{|c|c|c|c|}
\hline $\begin{array}{l}\quad 2013 \\
\text { Smulders, E., } \\
\text { Enkelaar, L., } \\
\text { Schoon, Y., } \\
\text { et al. }\end{array}$ & $\begin{array}{l}\text { Falls prevention in } \\
\text { persons with intellectual } \\
\text { disabilities: Development, } \\
\text { implementation, and } \\
\text { process evaluation of } \\
\text { a tailored multifactorial } \\
\text { fall risk assessment and } \\
\text { intervention strategy }\end{array}$ & DI & Focus grup \\
\hline $\begin{array}{l}\qquad 2014 \\
\text { Cocks, E.; } \\
\text { Thoresen, S.; } \\
\text { Williamson, } \\
\text { M., et al. }\end{array}$ & $\begin{array}{l}\text { The individual supported } \\
\text { living (ISL) manual: } \\
\text { A planning and } \\
\text { review instrument for } \\
\text { individual supported } \\
\text { living arrangements for } \\
\text { adults with intellectual } \\
\text { and developmental } \\
\text { disabilities. }\end{array}$ & Adultos con DI & $\begin{array}{l}\text { Individual supported } \\
\text { living (ISL) }\end{array}$ \\
\hline $\begin{array}{l}\quad 2015 \\
\text { Boddy, L.M., } \\
\text { Downs, S., } \\
\text { Kowles, Z.R. } \\
\text { et al. }\end{array}$ & $\begin{array}{l}\text { Physical activity and play } \\
\text { behaviours in children } \\
\text { and young people with } \\
\text { intellectual disabilities: } \\
\text { A cross-sectional } \\
\text { observational study }\end{array}$ & $\begin{array}{l}\text { Niños y } \\
\text { adolescents } \\
\text { con DI }\end{array}$ & $\begin{array}{l}\text { Systematic } \\
\text { observation } \\
\text { techiques }\end{array}$ \\
\hline
\end{tabular}

\section{DISCUSIÓN Y CONCLUSIONES}

La evaluación en personas con DI, y especialmente con DI moderada o severa, presenta dificultades a la hora de aplicar ciertos cuestionarios o pruebas psicométricas en las investigaciones, por ello encontramos estudios donde las personas que puntúan los instrumentos son los cuidadores o tutores de la persona. Para ello, un tipo de instrumentos efectivos serán aquellos fundamentados en la observación, bajo un marco metodológico estricto, tal y como afirma Anguera (1989). En este caso el análisis observacional permitiría al profesional obtener información de aquellas conductas o elementos que considera importantes, que se escapan a la atención y difícilmente pueden traducirse en palabras (Anguera 1989).

En los resultados obtenidos en la primera revisión sistemática (ver Tabla 3) destacan los estudios referentes a intervenciones vinculadas con danza, danza terapia u otro tipo de terapias creativas en población infantil y juve- 
nil en DI. En cambio, solamente uno de los estudios se centra en población adulta con DI coincidiendo con las aportaciones de Meredith Ritter y Kathryn Low (1996). Además, observamos que dentro de la población con DI destacan aquellos estudios dirigidos a población del espectro del autismo en danza, tal y como observamos en el estudio de Sabine Koch, Teresa Kunz, Sissy Lykou y Robin Cruz (2014).

Referente a la primera revisión, observamos que tres de los estudios utilizan el dibujo como instrumento de evaluación y/o diagnóstico. Además destacan tres artículos que se focalizan en instrumentos de observación. Estos estudios son los que evalúan elementos más similares a los cambios de movimiento, por lo tanto consideramos que un instrumento de tipo observacional sería adecuado para evaluar lo qué sucede en la sesión, referente a los cambios de movimiento de los participantes.

La segunda revisión sistemática (ver Tabla 4), muestra diferentes estudios vinculados a la DI donde observamos la utilización de instrumentos muy diversos. Pero en esta revisión no se han encontrado estudios vinculados específicamente en el ámbito de la danza. En estos estudios, encontramos más presencia de evaluación en población adulta con DI y estos están vinculados a temáticas relacionadas con la calidad de vida, protocolos de actuación con este tipo de población, comorbilidad con trastornos emocionales, así como el análisis de conductas, antes o después de una posible intervención, pero no lo que sucede durante la evaluación y tratamiento. Todos estos estudios nos permiten extraer elementos importantes a la hora de determinar un posible instrumento útil para población con DI.

Con los resultados obtenidos de las dos revisiones sistemáticas, así como el marco teórico del estudio, determinamos las características a tener presentes para la propuesta de protocolo para la creación de un instrumento de evaluación que nos permita evaluar los cambios de movimiento en el ámbito de la danza en población con DI:

- Revisión bibliográfica, documentación, clarificación de conceptos, marco teórico y metodología.

- Identificación de los elementos considerados principales para la realización del instrumento: Definiciones precisas de los elementos. 
Sistema de categorización. Determinar el número de dimensiones. Determinar las características de la observación.

- Realizar un seguimiento de la creación del instrumento con asesoramiento de un experto en metodología y estadística.

- La validez del instrumento: Revisión exhaustiva de los conceptos para determinar las variables utilizadas y utilización de la técnica de jueces expertos.

- La fiabilidad del instrumento: Análisis estadístico Kappa de Cohen.

- Antes de utilizar el instrumento, realizar un análisis exploratorio aplicando el instrumento en población con DI.

Concluimos que para complementar la información obtenida con el DFH, sería interesante utilizar un instrumento observacional, el cual nos permitiría conocer lo que sucede en la sesión respecto al movimiento. También detectamos la existencia de pocos estudios que vinculen la danza en población adulta con DI. Además, observamos que no existe ningún instrumento que evalúe cambios de movimiento en población con DI.

\section{Referencias}

AAIDD (American Association on Intellectual and Developmental Disabilities). 2011. Discapacidad intelectual: Definición, clasificación y sistemas de apoyo. Traducción de Miguel Ángel Verdugo Alonso. Madrid: Alianza

Anguera Argilaga, María Teresa. 1989. Metodología de la Observación en las ciencias humanas. Madrid: Cátedra

Ballesta Cervantes, Ana María, Onil Vizcaíno y Eva Cristina Mesas Escobar. 2011. "El Arte como un lenguaje posible en las personas con capacidades diversas". Arte y Políticas de Identidad 4: 137-52

Barnet López, Silvia y Myriam Guerra Balic. 2012. "La danza como recurso para fomentar el bienestar emocional". Póster presentado en el /l Congreso Nacional de Investigación en Danza, Barcelona

Barnet López, Silvia, Susana Pérez Testor y Myriam Guerra Balic. 2013. "El autoconcepto en la Danza Movimiento Terapia con personas adultas con discapacidad intelectual: Estudio de un caso grupal". Póster presentado en el III Congreso Nacional de Terapias Creativas", Vitoria

Fischman, Diana. 2008. "Relación terapéutica y empatía kinestésica”. Pp. 81-96 en La vida es danza: El arte y la ciencia de la Danza Movimiento Terapia, Hilda Wengrower y Sharon Chaiklin, coords. Barcelona: Gedisa

Hecox, Bernadette, Ellen Levine \& Diana Scott. 1975. "A report of the use of dance in physical rehabilitation". Rehabilitation Literature 36: 11-15 
Hurley, Anne, Daniel Tomasulo \& Albert Pfadt. 1998. "Individual and group psychotherapy: Approaches for persons with mental retardation and developmental disabilities". Journal of Developmental and Physical Disabilities 10: 365-86

Jané Ballabriga, Maria Claustre. 2004. "Diagnòstic dual en la deficiència mental". Suports 8 : 42-58

Koch, Sabine, Teresa Kunz, Sissy Lykou \& Robin Cruz. 2014. "Effects of dance movement therapy and dance on health-related psychological outcomes: A meta-analysis". The Arts in Psychotherapy 41: 46-64

Koppitz, Elizabeth. 2000. El dibujo de la figura humana. Buenos Aires: Guadalupe

Laban, Rudolf. 1987. El dominio del movimiento. Madrid: Fundamentos.

Martín Borràs, María Carmen, Susana Pérez Testor y Myriam Guerra Balic. 2009. "La danza y la expresión corporal en el desarrollo emocional de la adolescente saharaui: estudio piloto". En Inteligencia emocional, 20 años de investigación y desarrollo: I/ Congreso Internacional de Inteligencia Emocional, Santander: Fundación Botín

Pérez-Testor, Susana y Anna Griñó Roca. 2014. "Proyecto DEC Danza Esquema Corporal: Dibujo de la figura humana con estudiantes adolescentes que practican danza contemporánea". En III Congreso Internacional de Danza, Investigación y Educación: Género e Inclusión Social, Málaga. En prensa.

Pérez-Testor, Susana, Xavier de Blas Foix, Silvia Barnet López y Elisa Guerra Bálic. 2012. "Initial assessment and exclusion criteria of the human figure drawing with university students in Taiwan". Pp. 374-7 en Together for physical education. Barcelona: Universitat Ramon Llull

Pérez-Testor, Susana, Montse Sanahuja-Maymó y Elisa Guerra Bálic, Eloisa Martínez Torregrosa, Núria Massó Ortigosa y Lluís Costa Tutusaus. 2009. "Impact of a creative dance class on intellectual and affective development in Down Syndrome adolescents". Pp. 12930 en IADMS 19th Annual Meeting: Abstracts. The Hague

Ritter, Meredith \& Kathryn Low. 1996. "Effects of dance/movement therapy: A meta-analysis". The Arts in Psychotherapy 23: 249-60

Rogers, Susan. 1977. "Contributions of dance therapy in a treatment program for retarded adolescents and adults". Art Psychotherapy 4: 195-7

Tipple, Blanche. 1975. "Dance therapy and education". Journal of Leisurability 2(4): 9-12

Verdonschot, Manon M.L, L.P. de Witte, E. Reichrath, W.H.E. Buntinx \& L.M.G. Curfs. 2009. "Impact of environmental factors on community participation of persons with an intelectual disability: A systematic review". Journal of Intellectual Disability Research 53: 55-64 